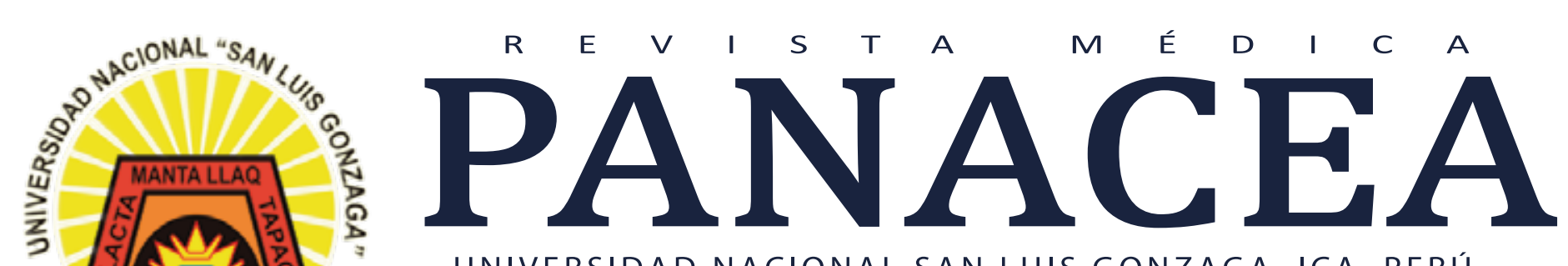

UNIVERSIDAD NACIONAL SAN LUIS GONZAGA. ICA, PERÚ

FACULTAD DE MEDICINA HUMANA "DANIEL ALCIDES CARRIÓN"

p-ISSN 2223-2893

e-ISSN 2225-6989

Volumen 9 Número 3

PUBLICACION CUATRIMESTRAL

Setiembre - Diciembre

2020

\title{
ARTÍCULO DE REVISIÓN: \\ OBESIDAD COMO FACTOR DE RIESGO PARA MORTALIDAD POR COVID-19.
}

OBESITY AS A RISK FACTOR FOR MORTALITY FROM COVID-19.

\section{AUTORES:}

LEVEAU BARTRA HARRY

ORISON ARMANDO LEVEAU BARTRA

JHON RODRIGO AUSEJO GALARZA

IVAN MARCO CÓRDOVA TELLO

JOSÉ LUIS CÓRDOVA TELLO

CHÁVEZ NAVARRO JUAN

GENG OLAECHEA, LUZ E.

INDEXADA EN:

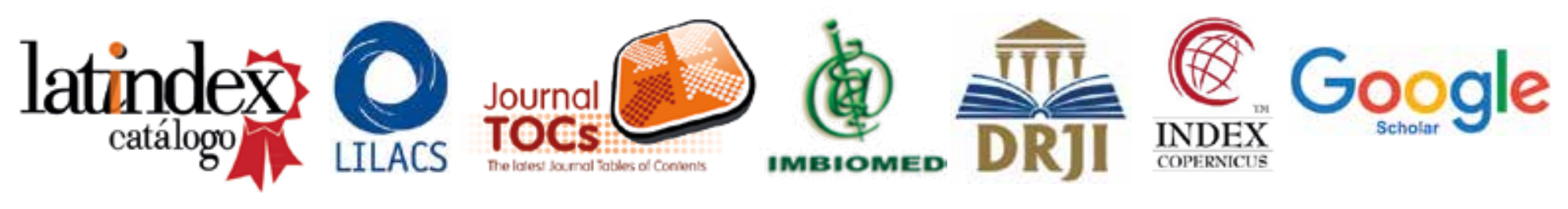

revistas.unica.edu.pe 


\title{
OBESIDAD COMO FACTOR DE RIESGO PARA MORTALIDAD POR COVID-19. OBESITY AS A RISK FACTOR FOR MORTALITY FROM COVID-19.
}

\begin{abstract}
Leveau Bartra Harry ${ }^{1, a}$, Orison Armando Leveau Bartra ${ }^{2, a}$, Jhon Rodrigo Ausejo Galarza ${ }^{1, b}$, Ivan Marco Córdova Tello ${ }^{1, b}$, José Luis Córdova Tello ${ }^{1, b}$, Chávez Navarro Juan R,c, Geng Olaechea, Luz E.,d.
\end{abstract}

\author{
1. Universidad Privada San Juan Bautista. Filial-Ica. \\ 2. Universidad Nacional de Ucayali \\ 3. Universidad Nacional Federico Villarrea \\ 4. Zegel IPAE
}

\author{
Cirujano General \\ b. Médico Cirujano \\ c. Licenciado en Psicología \\ d. Licenciada en Educación
}

DOI: https://doi.org/10.35563/rmp.v9i3.372

\section{Correspondencia: \\ Harry Leveau Bartra (ORCID: 0000-0001-9632-7793)}

Correo: kahaisail4@hotmail.com
Contribuciones de autoría:

Conflicto de intereses: no existen conflictos de intereses del autor o autores de orden económico, institucional, laboral o personal.

Financiamiento:

Autofinanciado.

\section{Cómo citar:}

Leveau-Bartra

Leveau-Bartra

Ausejo-Galarza JR, Ivan Mar Córdova-Tello IM, José Luis

Córdova-Tello

Chávez-Navarro

Geng-Olaechea

Obesidad como factor de riesgo para mortalidad por COVID-19. Rev Méd Panacea. 2020;9(3) 184-188. DOl:

https://doi.org/10.35563/r mp.v9i3.372

Recibido: 24 - $10-2020$

Aceptado: 08 - 11 - 2020

Publicado: 02 - 12 - 2020

\section{RESUMEN}

Objetivo: Valorar la magnitud del riesgo de la obesidad en la mortalidad por COVID-19. Materiales y métodos: Estudio es de tipo observacional, descriptiva, transversal, retrospectiva. La población corresponde a estudios realizados sobre obesidad como factor de riesgo para mortalidad por COVID-19, siendo encontrado en la base de datos de PubMed, Lilacs y Scielo 45 estudios de las que se pudieron reproducir un total de 07 que cumplieron los criterios de reproducibilidad, especificidad y rigor científico. Resultados: la OR global en el modelo de efectos fijos es de $1.28($ IC95\%= $1.06-1.55)$ y en el modelo de efectos aleatorios es de 1.30 (IC95\%= 1.03 - 1.64) de los 7 estudios analizados sobre 7876 pacientes. Los estudios analizados presentan $24.6 \%$ de variación en OR atribuible a la heterogeneidad, siendo prácticamente baja la heterogeneidad. El tener obesidad incrementa la probabilidad de morir por COVID-19 en 0.28 (28.3\%) veces en comparación que si no estaría presente esta condición patológica durante el padecimiento de la COVID-19. Conclusiones: La obesidad es un factor de riesgo que incrementa la probabilidad de muerte por COVID-19

Palabras clave: Obesidad, mortalidad, coronavirus.

\section{ABSTRACT}

Objective: To assess the magnitude of the risk of obesity in mortality by COVID-19. Materials and methods: Study is observational, descriptive, transversal, retrospective. The population corresponds to studies carried out on obesity as a risk factor for mortality by COVID-19, being found in the database of PubMed, Lilacs and Scielo 45 studies from which it was possible to reproduce a total of 07 that fulfilled the criteria of reproducibility, specificity and scientific rigor. Results: The global OR in the fixed effect model is 1.28 (IC95\%= $1.06-1.55)$ and in the random effect model is $1.30($ IC95\%= $1.03-1.64)$ of the 7 studies analyzed on 7876 patients. The studies analyzed present $24.6 \%$ variation in OR attributable to heterogeneity, with the heterogeneity being practically low. Having obesity increases the probability of dying from COVID-19 by 0.28 (28.3\%) times compared to otherwise this pathological condition would be present during COVID-19. Conclusions: Obesity is a risk factor that increases the probability of death from COVID-19

Keywords: Obesity; Mortality; Coronavirus. 


\section{INTRODUCCIÓN}

Según la Organización Mundial de la Salud-OMS $(1,2)$ define como sobrepeso u obesidad en los adultos como: sobrepeso: Índice de Masa Corporal (IMC) igual o más de 25 y obesidad: IMC igual o más de 30. La obesidad desde 1975 casi se ha triplicado a nivel mundial. Más de 1.900 millones de personas de más de 18 años sufrían sobrepeso y de ellos 650 millones sufren de obesidad en el 2016. Esto se debe, al crecimiento del mercado del consumo paulatino de los denominados productos superprocesados que va reemplazando a alimentos naturales o procesados mínimamente en la dieta de la población, en esta realidad, la OMS(3) especifica que aproximadamente el $58 \%$ de todos los casos de diabetes, el $21 \%$ de la cardiopatía isquémica y del $8 \%$ al $42 \%$ de algunos cánceres son debido a un IMC alto. En este contexto una nueva enfermedad causada por un virus denominado COVID-19 azota la humanidad desde diciembre del 2019, provocando un incremento de fallecimientos en gran parte del mundo, lo que provocó que la comunidad científica vuelque su mirada en esta enfermedad, sobre todo buscando las entidades nosológicas que incrementan la posibilidad de muerte, siendo identificados algunos como las edades avanzadas, las comorbilidades y en esta última es también incluida la obesidad. El INEI(4) indica que en el Perú el IMC medio de las mujeres es de 26,8 y de los hombres de 25,7 a estos datos se suma lo indicado por Morales(5) quien concluye que la situación epidemiológica de nuestro país permite medir la severidad de la obesidad. Esto se debe a que la obesidad afecta a 7 de cada 10 adultos y 3 de cada 10 niños. Conociendo que en el Perú la Obesidad no deja de ser un problema de salud es que analizamos en este artículo los hallazgos de la obesidad como factor que incrementa la probabilidad de muerte por COVID-19 en un estudio de metanálisis a fin de obtener un valor de riesgo según los resultados de investigaciones realizadas en diferentes lugares con lo que se incrementa la potencia de la investigación en este tema.

\section{MATERIAL Y MÉTODO}

La población corresponde a estudios realizados sobre obesidad como factor de riesgo para mortalidad por COVID-19, siendo encontrado en las bases de datos PubMed, Lilacs y Scielo, 45 estudios de las que se pudieron reproducir un total de 07.

\section{Criterios de inclusión:}

- Estudios que pueden ser reproducibles por los datos que presentan.

\section{Criterios de exclusión:}

- Aquellos estudios que no cuenten con el diseño y el rigor científico correcto.

La muestra. Constituida por 07 estudios cumplieron con los criterios de reproducibilidad, especificidad y rigor científico.

\section{Unidad de análisis}

Estudio sobre obesidad y riesgo de mortalidad por COVID-19

\section{Análisis de la información}

Las investigaciones fueron procesadas en Epidat 3.1. a fin de determinar el ODDS Ratio ponderado según tamaño de muestra de cada estudio tomando en cuenta la heteregenidad con la prueba de Q de DerSimonian y Lard, se obtiene y analiza el gráfico de Galbraith, así mismo se obtiene el gráfico de Forestplot y Funnelplot.

\section{RESULTADOS}

Artículo de revisión mide la Odds ratios con un nivel de Significancia de $95.0 \%$ habiéndose encontrado 7 investigaciones reproducibles.

\section{HETEROGENEIDAD}

Prueba de heterogeneidad de Dersimonian y Laird's

Estadístico Q (Chi cuadrado)= 7.956

$\mathrm{gl}=6$

Valor $\mathrm{p}=0.241$

$12=24.6 \%$ (Variación en OR atribuible a la heterogeneidad)

La prueba de heterogeneidad de Dersimonian y Laird's (Chi cuadrado) es de 7.96 con un valor de $p=0.24$ que como coeficiente de heterogeneidad indicaría que los estudios son homogéneos $(p>0.05)$ pues tendríamos un $24 \%$ de probabilidad de error al decir que son heterogéneos, así mismo al calcular el índice de inconsistencia o 12 este es de $24.6 \%$ que indica que prácticamente tiene baja heterogeneidad.

\section{Estadístico de heterogeneidad}

\section{Estimador}

Varianza entre estudios

$$
0.0235
$$

Varianza intra-estudios

0.0640

Coeficiente RI

0.2688 (Proporción de varianza total debida a la varianza entre

Coef. variación entre estudios estudios)

0.6156

El coeficiente RI en este análisis es de $26.88 \%$ que es la proporción de la varianza total que es debida a la varianza entre estudios. De todos los estudios el de Leonidas(12) es que es más variable en relación a los demás pues el OR calculado en este estudio se aleja de los valores encontrados en los 6 estudios restantes por lo que en el gráfico de Galbraith este estudio se encuentra fuera de las líneas paralelas y muy alejado de la línea central, la que se debería a la poca muestra empleada para este estudio, mientras que, el estudio de Sara(10) y de Meneses(6) son los estudios que fueron realizados en una muestra amplia con resultados muy similares.

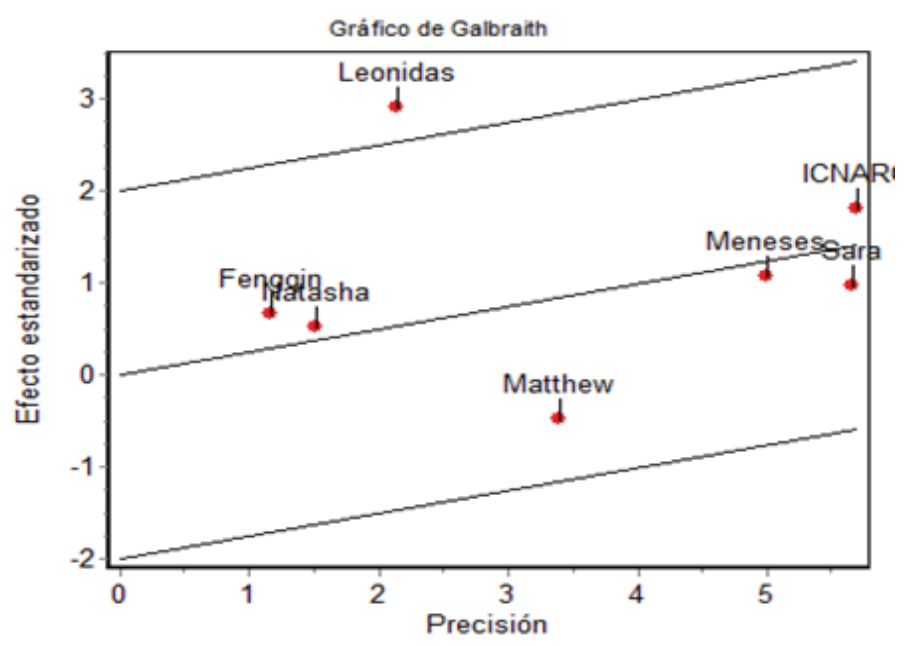


Al evaluar los resultados en el gráfico de L'Abblé se observa que solo el estudio de Matthew(7) se ubica en el lado de los controles, aunque no muy alejado de ella, y los restantes 6 estudios se ubican en el lado de los casos que indican que la obesidad es un factor que incrementa la mortalidad por COVID-19.

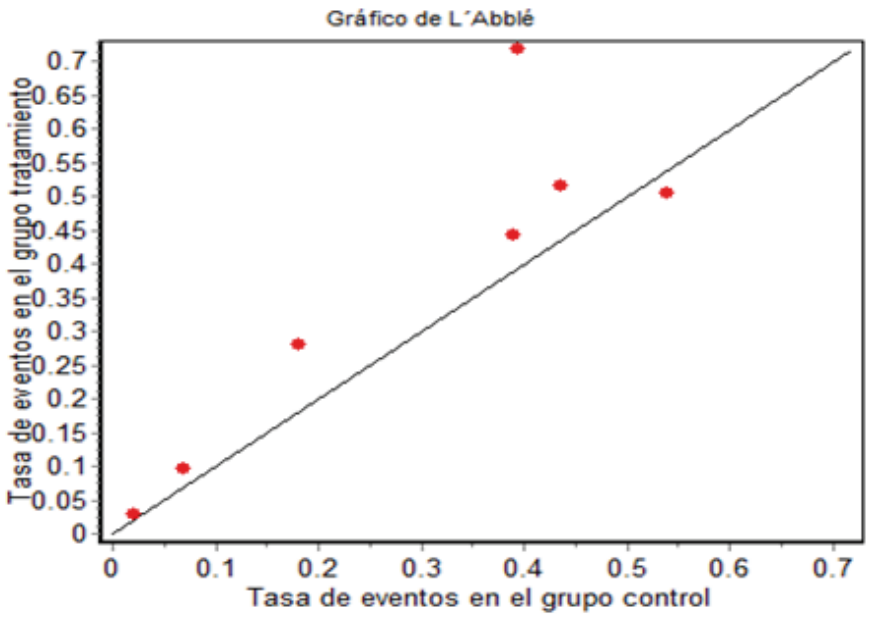

RESULTADOS INDIVIDUALES Y COMBINADOS

\begin{tabular}{|c|c|c|c|c|c|c|}
\hline \multirow[b]{2}{*}{ Estudio } & \multirow[b]{2}{*}{ Año } & \multirow[b]{2}{*}{$\mathbf{n}$} & \multirow[b]{2}{*}{ OR } & \multirow[b]{2}{*}{ IC(95.0\%) } & \multicolumn{2}{|c|}{ Pesos(\%) } \\
\hline & & & & & E. fijos & E. aleat. \\
\hline Meneses(6) & 2020 & 1152 & 1,237 & $0.837 \quad 1.831$ & 22.904 & 22.786 \\
\hline Matthew(7) & 2020 & 195 & 0,868 & $0.488 \quad 1.545$ & 105.599 & 131.293 \\
\hline ICNARC(8) & 2020 & 623 & 1,372 & $0.973 \quad 1.936$ & 296.520 & 265.928 \\
\hline Fengqin(9) & 2020 & 54 & 1,742 & 0.3289 .261 & 12.581 & 19.269 \\
\hline Sara(10) & 2020 & 5579 & 1,186 & 0.8391 .677 & 293.292 & 264.278 \\
\hline Natasha(11) & 2020 & 189 & 1,414 & $0.387 \quad 5.169$ & 20.905 & 31.367 \\
\hline Leonidas(12) & 2020 & 84 & 3,892 & 1.5619 .707 & 42.059 & 60.009 \\
\hline Efectos fijos & & 7876 & 1,283 & 1.0641 .547 & & \\
\hline Efectos aleatorios & & 7876 & 1,300 & $1.027 \quad 1.646$ & & \\
\hline
\end{tabular}

\section{FOREST PLOT}

\section{META-ANÁLISIS ACUMULADO (Efectos aleatorios)}

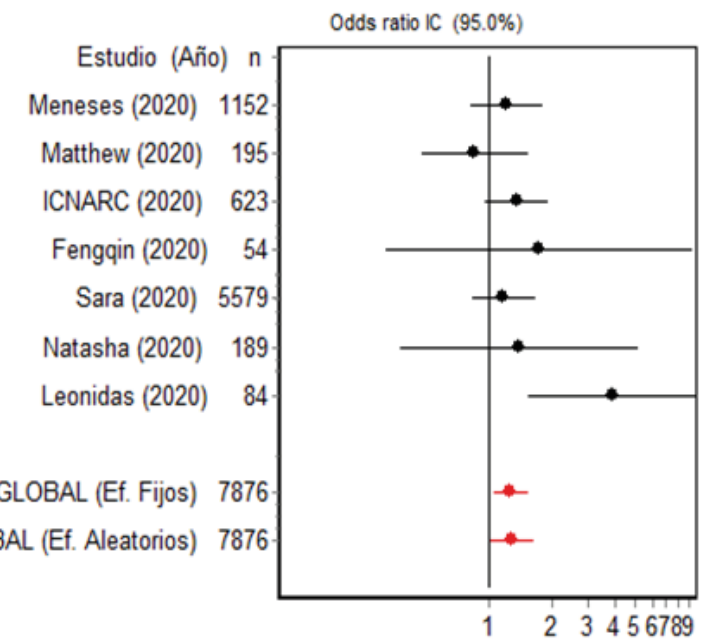

El gráfico de Forest plot muestra una OR consolidada de 1.283 $(\mathrm{IC} 95 \%=1.064-1.547)$ valores que no incluyen al 0 en el modelo de efectos fijos indicando que existe significancia estadística incrementando en un $28 \%$ la posibilidad de morir por COVID-19 si la condición del paciente es obesidad en comparación con los que no son obesos.

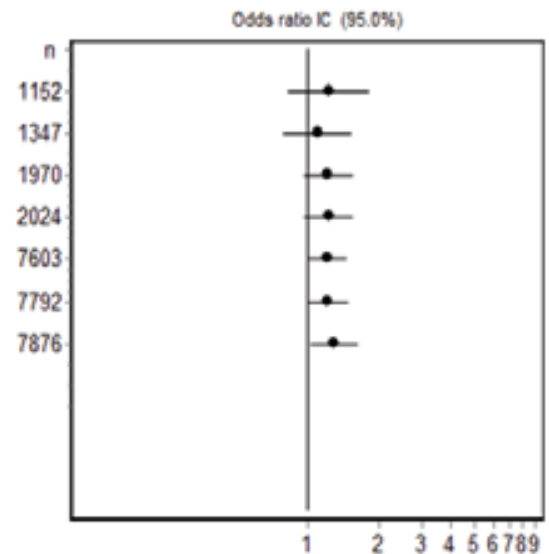

En el gráfico de OR acumulado muestra que en una metaregresión es el estudio de Matthew(7) la que desvía la OR hacia la línea media sin poder sobrepasarlo pues el estudio de Meneses tiene un gran peso (22.9) por la amplitud de su muestra y la especificad del intervalo de confianza, y para delante la OR queda estabilizada por el estudio de ICNARC(9) y Sara(10) que tienen una gran muestra y alta especificidad lo que le da un alto peso (29.65 y 29.33 respectivamente).

Al determinar el Sesgo de publicación, la Prueba de Begg tiene un Estadístico $Z=0.3004$ con un valor de $p=0.7639$ y en la Prueba de Egger cuyo Estadístico $t=$

0.9331 con 5 grados de libertad presenta un Valor de $p=$ 0.3936
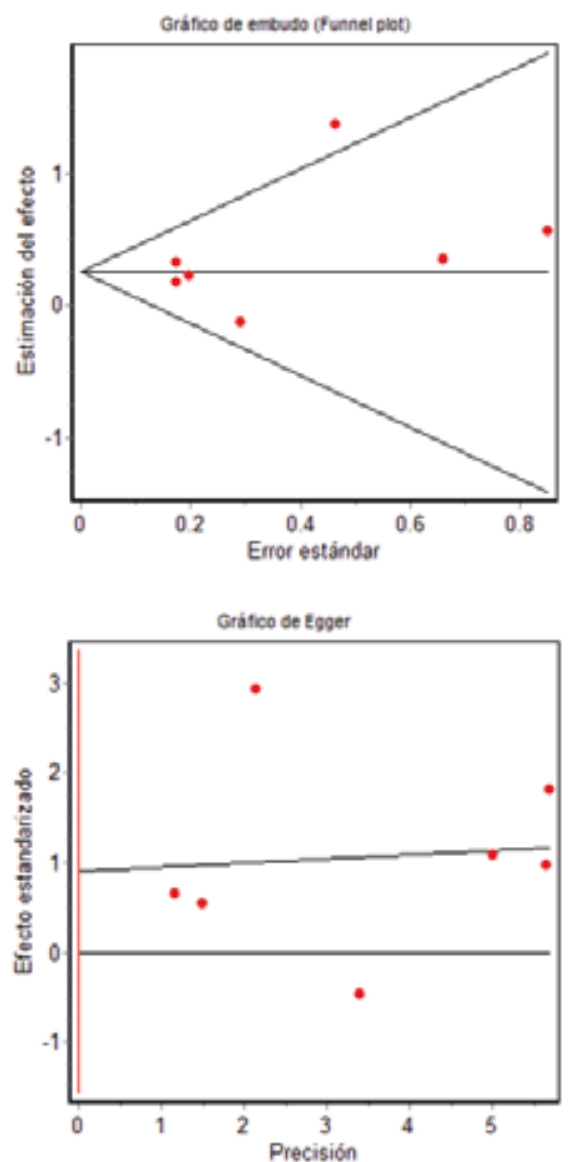
La prueba de Begg muestra el grado de sesgo en los estudios cuyo valor de p es de 0.7639 lo que indica que el sesgo no es significativo corroborado por la prueba de Egger que muestra una t de 0.9331 y un valor de $p$ de 0.3936 que mucho mayor a 0.05 . Ello se puede observar en el gráfico Funel plot pues los puntos se distribuyen a ambos lados de la line media con 3 estudios con alta especificidad como son los de Meneses(6), ICNARC(8) y Sara(10).

\section{ANÁLISIS DE SENSIBILIDAD}

\section{MODELO EFECTOS ALEATORIOS}

\begin{tabular}{lcclccc} 
& & & \multicolumn{4}{c}{ IC(95.0\%) } \\
Estudio & Año & $\mathbf{n}$ & OR & L. Inferior & L. Superior & Cambio relativo \\
Meneses & 2020 & 6724 & 1,3421 & 0,9803 & 18.376 & 3.24 \\
Matthew & 20207681 & 1,3676 & 1,0833 & 17.266 & 5.20 \\
ICNARC & 20207253 & 1,3019 & 0,9446 & 17.944 & 0.15 \\
Fengqin & 20207822 & 1,2994 & 1,0050 & 16.801 & -0.05 \\
Sara & 20202297 & 1,3638 & 0,9919 & 18.753 & 4.91 \\
Natasha & 20207687 & 1,3053 & 1,0047 & 16.958 & 0.40 \\
Leonidas & 20207792 & 1,2219 & 1,0090 & 14.798 & -6.01 \\
\hline GLOBAL & 7876 & 1,3000 & 1,0271 & 16.455 & \\
\hline
\end{tabular}

Gráfico de influencia

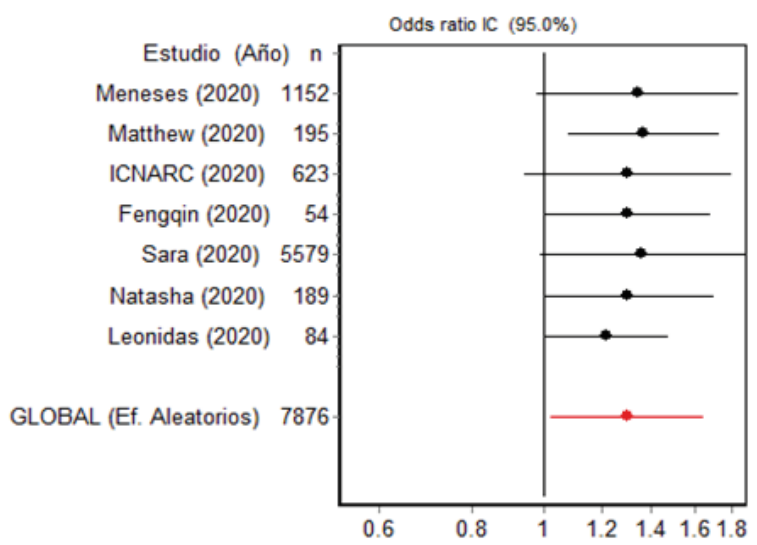

Es de notar que en el gráfico de influencia es el estudio de Leonidas la que más influye en el orden de las OR acumulados, pero por la robustez de los estudios esta modificación no es significativa indicando que la obesidad es un factor de riesgo que incrementa la probabilidad de muerte por la COVID-19, ello debido a que, la obesidad es el exceso de grasa acumulada por un balance positivo de energía determinado por cambios en la ingesta y el consumo.

\section{DISCUSIÓN}

Determinar en qué medida la obesidad incrementa el riesgo de muerte por COVID-19 es el objetivo de este metanálisis, en tal sentido se determinó que la OR consolidada de los 7 estudios es de 1.283 (IC95\%= 1.064 - 1.547), este hallazgo lo indica Petrova(13) en su estudio donde indica que los datos son sugestivo de que la obesidad incrementaría el riesgo de morir en los obesos jóvenes, mientras que Acosta(14) en su estudio indica que el sobre peso y la obesidad no incrementaría el riesgo de morir por cualquier enfermedad aunque en los jóvenes si incrementaría este riesgo, estos hallazgos aparentemente contradictorios se debería a que el riego es medido tanto en los pacientes con sobre peso y obesidad que sería distinto si el estudio se realice solo en obesos y ello porque Preciado(15) en su estudio indica que la obesidad conduce a una inmunodeficiencia resultante de la producción alterada de intermediarios metabólicos en las células grasas, y se han propuesto posibles mecanismos que están involucrados en el aumento de la susceptibilidad y la gravedad de las infecciones en pacientes obesos, principalmente alrededor de la leptina. Sin embargo, no existen estudios prospectivos que puedan aclarar la relación entre la obesidad y el desarrollo de enfermedades infecciosas. Pues como dice Ángeles(16) en la obesidad existen evidencias de un aumento de expresiones de receptores del tipo Toll (TLRs) en la célula adiposa, estos receptores tienen gran participación en las respuestas innatas. Así, la obesidad produce un síndrome de hipoventilación perjudicial en circunstancias que el paciente presente la COVID-19 que además tiene hipoperfusión diurna (eliminación de $\mathrm{CO} 2$ dificultosa); trastornos respiratorios del sueño (apnea del sueño). El síndrome de hipoventilación por obesidad (SHO) es conocido como síndrome de Pickwick. Algunas sujetos con SHO pueden presentar síntomas como los descritos por Charles Dickens "Los papeles póstumos del club Pickwick puesto de relieve en el estudio de la American Thoracic Society(17). Finalmente, la obesidad tiene un componente genético multigénico (alrededor de 60-70\%) y un componente ambiental (alimentación, sedentarismo, flora del intestino). Del mismo modo, la obesidad se considera una condición proinflamatoria. Los niveles elevados de proteína $C$ reactiva (PCR) están asociados con un mayor riesgo de infarto cardiaco, accidente cerebrovascular y enfermedad arterial periférica, citado en Rincon(18).

Los estudios de casos y controles realizados sobre la temática desarrollada son múltiples, sin embargo, solo algunos pueden ser reproducibles por los escasos datos que indican en el desarrollo de dichos estudios, en este estudio se revisaron 45 estudios y solo 7 pudieron ser reproducibles, por lo que se recomienda que los estudios de casos y controles deben mostrar datos de valores absolutos y porcentuales a fin de poder analizar más estudios la que incrementa la potencia de los resultados al tener más muestras analizadas.

\section{CONCLUSIONES}

- La OR global en el modelo de efectos fijos es de 1.283 (IC95\% = 1.064 - 1.547) y en el modelo de efectos aleatorios es de $1.3($ IC95\% $=1.027-1.646)$ de los 7 estudios analizados sobre 7876 pacientes.

- Los estudios analizados presentan $24.6 \%$ de variación en OR atribuible a la heterogeneidad, siendo prácticamente baja la heterogeneidad.

- El tener obesidad incrementa la probabilidad de morir por COVID-19 en 0.283 (28.3\%) veces en comparación que si no estaría presenta esta condición patológica durante el padecimiento de la COVID-19. 


\section{REFERENCIAS BIBLIOGRÁFICAS}

1. OMS. Obesidad y sobrepeso, datos y cifras 2020. Recuperado de: Obesidad y sobrepeso - World Health Organizationwww.who.int

2. Pajuelo J. La obesidad en el Perú. An Fac med. 2017;78(2):179-185.

DOI: http://dx.doi.org/10.15381/anales.v78i2.13214.

3. Organización Mundial de la Salud (OMS). Informe sobre la salud en el mundo 2002 - Reducir los riesgos y promover una vida sana. OMS, editor. World Health Organization; 2013. Citado en: Malo-Serrano M, Castillo N, Pajita D. doi.org/10.15381/anales.v78i2.13213

4. Instituto Nacional de Estadística e Informática, Encuesta Nacional de Demografía y Salud, Perú 2015. Recuperado de: proyectos.inei.gob.pe > endes

5. Morales L, Ruvalcaba J. La obesidad, un verdadero problema de salud pública persistente en México. JONNPR. 2018;3(8):643-654. DOI: 10.19230/jonnpr.2544

6. Menezes R, Rodrigues L, Martins L. Factores de riesgo de hospitalización y mortalidad por COVID-19 en el estado de Espírito Santo, Brasil. Am J Trop Med Hyg. 2020 Jul 16. doi: 10.4269/ajtmh.20-0483. Online ahead of print.

7. Matthew J, Matthew R, Darryl, S. Epidemiología, curso clínico y resultados de adultos críticos con COVID-19 en la ciudad de Nueva York: un estudio de cohorte prospectivo. Rev. Lancet 6 de junio de 2020; 395 (10239): 1763-1770. doi: 10.1016 / S0140-6736 (20) 31189-2. Epub 202019 de mayo.

8. ICNARC report on COVID-19 in critical care 04 April 20. Intensive Care National Audit and Research Centre. https://www.icnarc.org/About/Latest-News/ 2020/04/04/Report-On-2249-Patients-Critically-III-With-C ovid-19.

9. Fengqin $Z$, Ying $X$, Yanqiu W, Yi H, Feng W, Gang L, Kui L, Ronghui D, Cong W, y Wenzhen Z. La obesidad predispone al riesgo de mayor mortalidad en pacientes jóvenes con COVID - 19. J Med Virol . 21 de mayo de 2020: 10.1002 / jmv.26039. doi: 10.1002 / jmv.26039

10. Sara $T$, et al. Obesidad y mortalidad entre pacientes diagnosticados con COVID-19: Resultados de una organización sanitaria integrada. Annals of Internal Medicine 2020. https://doi.org/10.7326/M20-3742

11. Natasha P. La obesidad se asocia con un mayor riesgo de mortalidad entre los pacientes hospitalizados con COVID-19. Obesidad (Silver Spring) . 26 de junio de 2020: 10.1002 / oby.22941. doi: 10.1002 / oby.22941

12. Leonidas $P$, et al. La obesidad grave, el aumento de la edad y el sexo masculino se asocian de forma independiente con peores resultados hospitalarios y una mayor mortalidad hospitalaria en una cohorte de pacientes con COVID-19 en el Bronx, Nueva York. Metabolismo . 2020 julio; 108: 154262.

13. Petrova $D$, Salamanca-Fernández E, Rodríguez-Barranco $M$. Navarro-Pérez $P$, Jiménez Moleón J, Sánchez $M$. La obesidad como factor de riesgo en personas con COVID-19: posibles mecanismos e implicaciones. Aten Primaria. 2020 Aug-Sep; 52(7): 496-500. doi: 10.1016/j.aprim.2020.05.003

14. Acosta L, Peláez E. Mortalidad atribuible al sobrepeso y la obesidad en Argentina: comparación entre los años 2005 y 2009. R. bras. Est. Pop., Rio de Janeiro, v. 32, n.2, p. 277-292, maio/ago. 2015.

http://dx.doi.org/10.1590/S0102-30982015000000016
15. Preciado M, Sánchez K, Álvarez M, González L, Ramos $M$, Andrade J. Obesidad e infecciones. Rev. Médica MD. 2018 9(4):341-344pp Recuperado

de: www.revistamedicamd.com

16. Ángeles F, García, R, Maldonado M, Alarcón F, Cruz M. EI papel de la inmunidad innata en la obesidad. Salud pública Méx vol.54 no.2 Cuernavaca mar./abr. 2012. Recuperado de: www.scielo.org. $m x$ > scielo

17. Serie de información al paciente de la ATS (C2014 American Thoracic Society. Síndrome de hipoventilación por obesidad. Am J Respir Crit Care Med, Vol. 189, P15-P16, 2014.

18. Rincón A. Fisiopatología de la obesidad. Universidad Complutense de Madrid 2016. Recuperado de: 147.96.70.122 > Web > TFG > TFG > Memoria

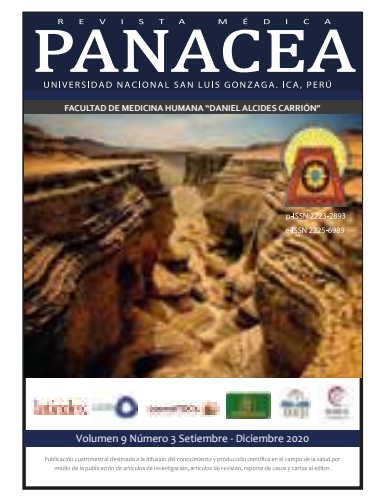

